\title{
Session 9: Therapeutic antibodies: Cancer
}

Friday 14th November 2008. Moderator: Zdenka Jonak

[09.00-09.30]

\section{Anticancer therapeutic antibody targeting cancer cell and tumor-associated activities \\ Yan $\mathrm{Wu}$ \\ ImClone Systems Incorporated, New York, USA}

Development of antibody-based cancer therapy has advanced substantially in research and clinical settings. The concept of antibody-directed therapeutic approaches was initially based on targeting a specific receptor or molecule that is expressed or functional in cancer cells. To achieve stronger antitumor effect, antibody combination and bispecific antibodies directed to cancer cells and other factors attributable to vascular or immune effector cells have been demonstrated to be effective in tumor treatment compared to single antibody based monotherapy. Conversely, an antibody directed to two or multiple molecules exerting functions in cancer cells, angiogenesis and other tumor-associated activities has been considered an attractive approach to achieving greater antitumor efficacy. Previously, we reported the antitumor activity of VEGFR-1 antibody through blocking the receptor-mediated functions in cancer and angiogenic cells. To date, the most difficult challenge to cancer intervention is the control of metastasis that accounts for most death of patients with cancer and the enhancement of antitumor immunity that is significantly weaken in most cancer patients. A number of growth factor/receptor signaling pathways including VEGFR-1 mediated signaling was reported to be active in immune cells and myeloid cells that regulate immunosuppression, angiogenesis, and metastasis. This talk will discuss on the potential of antibody-mediated antitumor activity through inhibition of metastasis-associated cells and immunosuppressive cells in tumor progression.
[09.30-10.00]

A variant IgG1 mediating improved ADCC

Ross Stewart, Michaela Levens, George Thom, Gülin

Güler-Gane, Robert Holgate, Chris Gee, Dave

Spencer, Carl Webster, Lutz Jermutus and John Lund

MedImmune Ltd., Milstein Building, Granta Park,

Cambridge CB21 6GH, UK

Ribosome display has been applied to human IgG1Fc in order to select for variants having improved binding to human Fc $\gamma$ RIIIa, the receptor expressed on human killer cells that mediates antibody dependent cellular cytotoxicity (ADCC). A library of $\mathrm{Fc} \gamma 1$ variants was generated using error-prone PCR. Multiple rounds of selection of an Fc $\gamma 1$ in ribosome display format were carried out using progressively decreased concentrations of soluble Fc $\gamma$ RIIIa to enrich for improved binders. Radioimmunoassay and alphascreen analyses of the aglycosylated IgG-Fc output revealed variants with improved binding to Fc $\gamma$ RIIIa relative to wildtype IgG-Fc. Subsequent expression in human (HEKEBNA) cells generated glycosylated IgGs that were assessed for binding to soluble Fc $\gamma$ RIIIa by ELISA.

One particular variant, 125 B01 showed a significant increase in the ability to trigger ADCC relative to wild-type antibody, and gave more equal levels of AD$\mathrm{CC}$ than wildtype IgG for each allotype of Fc $\gamma$ RIIIa, V158 and F158. Analysis of the oligosaccharide profiles of 125_B01 indicated increased levels of oligosaccharide chains lacking fucose relative to wildtype $\mathrm{IgG} 1$, for antibodies expressed in either HEK-EBNA or CHO cells. The increase in afucosylated oligosaccharide chains present in 125_B01 correlates with the replacement Phe-243 $\rightarrow$ Leu found in this variant. Residue 243 is one of many carbohydrate contact residues within the $\mathrm{C}_{H} 2$ domain of IgG (see below) and presumably can influence synthesis of nascent oligosaccharide chains of IgG-Fc within the Golgi apparatus. 


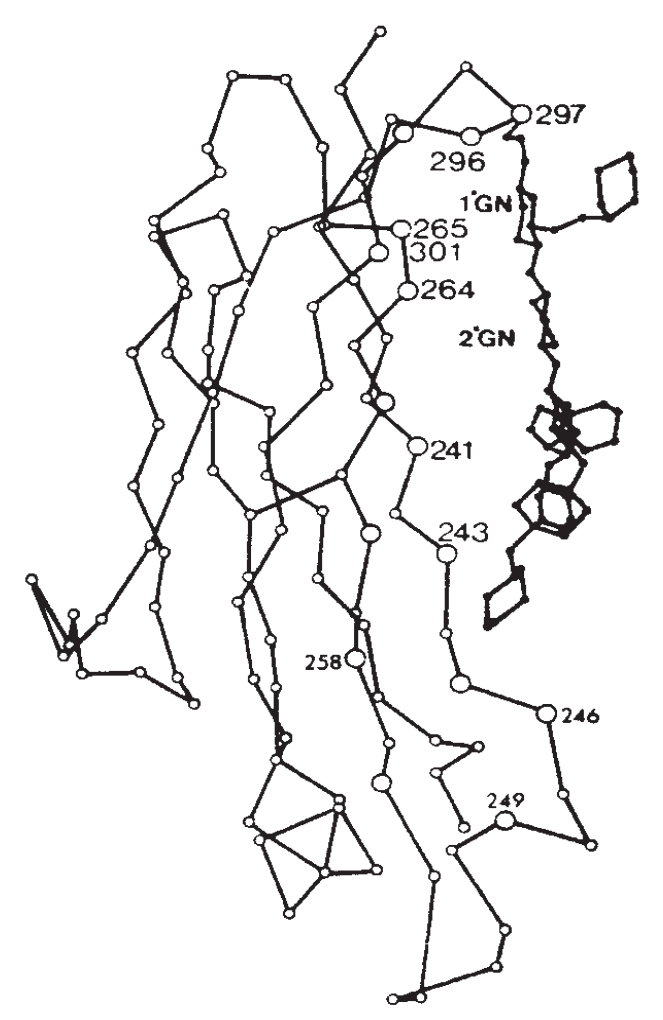

[10.00-10.30]

\section{Humanised anti-MUC1 antibody (AS1402) for the treatment of advanced breast cancer \\ Nigel Courtenay-Luck \\ Antisoma Research Ltd, Chiswick Park Building 5, 566 Chiswick High Road, London W4 5YF, UK}

Targeted therapy is now a well established therapeutic modality in clinical oncology, with many new drugs having been approved, both biologicals, such as monoclonal antibodies, and new chemical entities. Numerous targets have been identified, which provide a good differentiation between tumour and healthy tissues. One such antigen is the aberrantly-glycosylated MUC1 protein which is over expressed in a high percentage of epithelial tumours, such as breast, ovarian, lung, colon, pancreatic and prostate.

This presentation describes the preclinical aspects of targeting this antigen with the humanised anti-MUC1 antibody, AS1402, (previously known as huHMFG1, $\mathrm{R} 1550$ ). Results of a dose-escalating phase I clinical trial in breast cancer patients with advanced disease will also be discussed, including pharmacokinetics, safety and efficacy. The design and rationale of a randomised multi-centre phase II study, combining AS1402 with an aromatase inhibitor, letrozole, in post-menopausal women with metastatic breast cancer will also be presented.

\section{[11.00-11.30]}

\section{Targeting Proteases for Antibody Therapeutic Intervention in Oncology}

Paul J. Kerr

Fusion Antibodies, Northern Ireland, UK

Antibody drugs represent a major class of antitumor therapeutics which have contributed to improved prognostic outcome for patients suffering from various forms of cancer. The characteristic target specificity, long half-life and reduced toxicity make antibodies attractive modalities for the treatment of tumors where long-term administration is often necessary. Traditionally, antibodies have been developed against targets such as membrane receptors or ligands where they evoke an agonistic / antagonistic response. However, it is also possible to exploit their binding characteristics to block enzymatic activities resulting in the same therapeutic endpoints as small molecule-based methodologies.

Many protease species have been identified as promoting tumorigenic processes such as angiogenesis, invasion and metastasis. These proteases are generally over-expressed in the microenvironment by tumor and tumor-associated cells, where they contribute to the remodelling of extracellular matrix and/or modulation of tumor-promoting stimuli. Although the development of small molecule inhibitors to proteases has been well documented, frequently the pharmacological application of these molecules is limited by their lack of specificity and intracellular accumulation. The application of antibodies with much higher specificity which bind and block substrate cleavage offers an alternative approach to the development of drugs that can block protease action associated with promoting aggressive tumor progression.

\section{[11.30-11.50]}

Development of a potent anti-TYRP1 fully human antibody for melanoma therapy

Xiaoqiang Kang

ImClone Systems Inc., 180 Varick Street, New York, NY 10014, USA

TYRP1 (tyrosinase-related protein 1) is a melanoma antigen expressed in melanosomes and on the surface of melanoma cells. Previous studies have shown that 
mouse antibodies to TYRP1 localized to melanomas in vivo and inhibited tumor growth and metastasis. Here, we describe the characterization of a novel fully human anti-TYRP1 MAb (20D7) generated by immunizing HuMAb mice (Medarex). 20D7 recognized recombinant and native human TYRP1 by Western blotting and ELISA, and native TYRP1 in melanoma cells as determined by flow cytometry analysis. The binding affinity to human TYRP1 for the human MAb was in the low $\mathrm{nM}$ range as determined by surface plasmon resonance kinetics. 20D7 can bind to human and mouse Fc receptor and induce a strong Antibody-Dependent Cell-Mediated Cytotoxicity (ADCC) and ComplementDependent Cytotoxicity (CDC) response against human melanoma cells in vitro. The antitumor activity of 20D7 was evaluated in human melanoma xenografts and metastatic models in athymic nude mice. Growth of subcutaneous and metastatic tumors was significantly suppressed by 20D7 treatment compared to human IgG control. Moreover, 20D7 in combination with Dacarbazine (DTIC) exhibited stronger antitumor activity than 20D7 or DTIC alone in both subcutaneous and metastatic models. The mechanism of action of 20D7 was investigated by using aglycosylated 20D7 or complement depleted mice. The results suggested that both ADCC and CDC play important roles for 20D7 mediated tumor regression. The results of these study suggest that human anti-TYRP1 MAb may be a potent therapeutic for the treatment of malignant melanoma.
[11.50-12.20]

\section{Structural Modifications Imparting Autophilic Properties to Antibodies}

Fan Gao, Thomas J. Kindt and Christopher A. Lipinski InNexus Biotechnology Inc, Scottsdale, AZ 85259, USA

Antibody-Antibody association occurring via intermolecular interaction at the site of antigen binding is the hallmark of "autophilic" activity. This oligomerization of antibodies which occurs subsequent to antigen binding is typified in the well described mouse TEPC-family of anti-phosphocholine antibodies. Autophilic associations result in measureable kinetic changes, such as increased Bmax and lower Kd (increased avidity) as well as enhancement of certain effector functions such as CDC. Herein we describe a photo-oxidative antibody modification approach that selectively converts kappa light chain tryptophan residues to n-formylkynureine and induces structural changes imparting autophilic properties. These modifications include a measurable increase in hydrophobicity and, in several cases studied, imparted enhanced activity typical of autophilic interactions to the antibody. A structure-based hypothetical model of the modified molecule has been developed and tested by a variety of empirical assays and kinetic measurements.

[12.20-12.50]

KEYNOTE:

Monoclonal antibodies to the $\mathbf{T}$ cell receptor (TCRm) in cancer and infectious disease Jon Weidanz

Receptor Logic Ltd., Amarillo, Texas, USA

Abstracts not provided. 